\title{
Reproducing the Bolund experiment in wind tunnel
}

\author{
Yeow, T.S., Cuerva-Tejero A. and Perez-Alvarez, J. \\ IDR/UPM, E.T.S.I. Aeronáuticos، Universidad Politécnica de Madrid, E-28040 Madrid, Spain
}

\begin{abstract}
The Bolund experiment has been reproduced in a neutral boundary layer wind tunnel (WT) at scale 1:115 for two Reynolds numbers. All the results have been obtained for an incoming flow from the $270^{\circ}$ wind direction (transect $B$ in the Bolund experiment jargon). Vertical scans of the velocity field are obtained using non-time resolved two components particle image velocimetry. Time-resolved velocity time series with a three component hot-wire probe have been also measured for transects at 2 and $5 \mathrm{~m}$ height and in the vertical transects at met masts M6, M3 and M8 locations. Special attention has been devoted to the detailed characterization of the inflow in order to reduce uncertainties in future comparisons with other physical and numerical simulations. Emphasis is placed on the analysis of spectral functions of the undisturbed flow and those of the flow above the island. The result's reproducibility and trustworthiness have been addressed through redundancy measurements using particle image velocimetry, two and three components hot-wire anemometry. The bias in the prediction of the mean speed is similar to the one reported during the Bolund experiment by the physical modellers. However, certain reduction of the bias in the estimation of the turbulent kinetic energy is achieved. The WT results of spectra and cosprectra have revealed a behaviour similar to the full-scale measurements in some relevant locations, showing that WT modelling can contribute to provide valid information about these important structural loading factors.
\end{abstract}

\section{KEYWORDS}

atmospheric boundary layer; wind tunnel simulation; complex terrain; wind energy

\section{Correspondence}

Yeow, T.S., IDR/UPM, E.T.S.I. Aeronáuticos, Universidad Politécnica de Madrid, E-28040 Madrid, Spain.

E-mail: teeseong.yeow@upm.es

\section{INTRODUCTION}

Wind tunnel (WT) simulations have been a crucial part of the two main collaborative studies on neutral boundarylayer flow over low hills with gentle slopes and over steep three-dimensional hills: the Askervein hill project ${ }^{1}$ and the Bolund experiment ${ }^{2}$, respectively. In both cases, the research community has mainly focused on the validation of different types of numerical models [developed to predict the mean wind field and, depending on the type of model, the turbulent kinetic energy (TKE) and even the components of the Reynolds stress tensor], such as linear models, ${ }^{3,4}$ Reynolds-averaged Navier-Stokes (RANS), ${ }^{4-6}$ large-eddy simulation (LES) ${ }^{4,7,8}$ or hybrid RANS/LES approaches. ${ }^{9}$ Very limited experiences have been documented on the modelling of spectra of the velocity components on Askervein, ${ }^{10}$ where rapid distortion theory is applied for such purpose, and none for the case of Bolund.

The related WT tests have received, comparatively, less attention in terms of the number of dedicated publications and with relation to the extension of the specific WT simulation analysis itself. In the context of the Askervein hill project, WT measurements have been analysed in the studies by Teunissen et al. ${ }^{11}$ and Stock and Bowen ${ }^{12}$ and have been compared with numerical models in the study by Walmsley and Taylor. ${ }^{3}$ Some comparisons between rapid distortion theory and full-scale results of velocity spectra on Askervein are documented in the study by Mann. ${ }^{10}$ In the case of Bolund, WT simulations have been reported in the studies by Bechmarn er al ${ }^{4}$, Conan $^{13}$ and Yeow et al. ${ }^{14,15}$

The Bolund experiment was initiated by RIS $\varnothing$-DTU in 2007, and it is already considered as the new test case for benchmarking of numerical and physical modelling of complex terrain flows in neutral conditions. This experiment was initially planned as a blind comparison of more than 50 computational models (linear, RANS and LES) and also incorporated physical simulations in a WT and in a water channel. Bolund is a hill of about $150 \times 75 \times 12 \mathrm{~m}$, surrounded by water with a long 

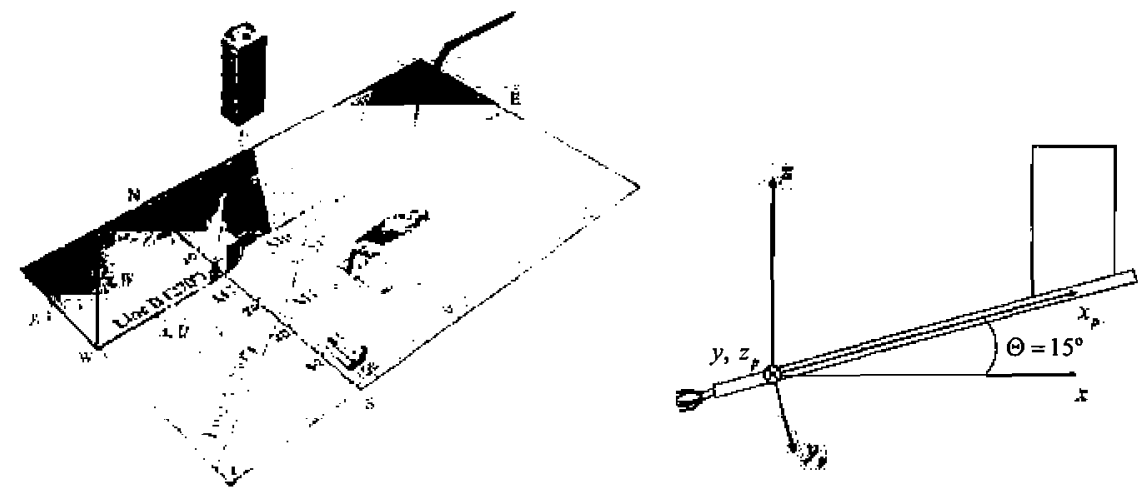

Figure 1. Left: experimental set-up. The met masts are indicated as $\mathbf{M}_{i}, i=1, \ldots, 8$. Right: mounting of the 3CHW probe 55P91 and its support. $\left[x_{p}, y_{p}, z_{p}\right]$ is the DANTEC probe reference system, ${ }^{20}$ and $[x, y, z]$ is the Bolund reference system. Note that the axis of the reference systems have not been plotted at the $(0,0)$ point which is located where lines $A$ and $B$ cross.

uniform fetch, for most of the upstream directions of interest. Only neutrally stratified atmospheric conditions fulfilling $\left|L_{\mathrm{Mo}}\right|>250 \mathrm{~m}$, where $L_{\mathrm{MO}}$ is the Monin-Obukhov length, were selected in the blind experiment. The small size of the hill allows to neglect Coriolis effects (the Rossby number is $R o=667$ ). ${ }^{16}$ The Reynolds number, $\operatorname{Re}_{h}=U_{h} h \nu^{-1}$, on the basis of the wind speed at $z=h$ ( $h$ is maximum height of the island), is in the range $4.25 \times 10^{6}<\operatorname{Re}_{h}<10.2 \times 10^{6}$, with $v$ as the kinematic viscosity.

One of the main geometric characteristics of Bolund is the escarpment facing approximately the wind directions 200 295 (Figure 1). The escarpment height varies slightly in the interval 200-295-, being roughly equal to the maximum height of the island $(11.73 \mathrm{~m})$. From now on, a full-scale value $h=11.73 \mathrm{~m}$ will be considered for reference purposes. Considering the $270^{\circ}$ wind direction as an example, the geometry of Bolund guarantees that flow detaches at the edge, provided a sufficiently large Reynolds number. The long (in the mean flow direction) flat top ensures re-attachment of the flow on the island. For this direction, the lee side of the hill presents a slope with a maximum inclination around $-40^{\circ}$, leading to intermittent re-circulation patterns (as it is shown in the succeeding text) such as in the lee side of Askervein. ${ }^{7}$ The existence of detachment at the escarpment has been verified by full-scale measurements of intermittent re-circulation patterns for the $239^{\circ}$ wind direction ${ }^{2}$ and $270^{\circ}$ wind direction. ${ }^{17}$ This has been also visualized by means of two components particle image velocimetry (PIV) in WT. ${ }^{14}$

The inflow full-scale roughness Reynolds number based on the friction velocity at $h_{0}=5 \mathrm{~m}, u_{* 05}=0.47 \mathrm{~ms}^{-1}$ (this friction velocity is defined in Section 2), and the aerodynamic roughness length, $z_{0}$, at the reference position M0 $\left(z_{0}=6 \times 10^{-4} \mathrm{~m}\right)$ is $u_{* 05} z_{0} \nu^{-1}=18.7$. 2 The corresponding value on the island is quite well above the previous value because of its rougher surface compared with the fetch. Therefore, since $u_{* 05} z_{0} v^{-1}>5$, the full-scale fetch boundary layer (BL) corresponds to a fully aerodynamic rough regime, clearly maintained on the island. ${ }^{16}$ The Jensen number based on the maximum height of the island is $h / z_{0}=1.96 \times 10^{4}$.

The main preliminary conclusions on the performance of the computational fluid dynamics models were outlined in Bechmann et al., ${ }^{4}$ where it was concluded that linear models presented the largest errors, since many of the assumptions of such models were violated because of the high complexity of Bolund topography. The blind experiment revealed unsuspected high speed-up errors in the LES models and allowed to identify RANS models with two closure equations as the most successful ones when predicting both the speed-up and the modification of TKE produced by the topography. Further computational simulations have been carried out after the publication of full-scale measurements. These new studies have been focused on the weak points identified during the blind experiment, such as the implementation of boundary conditions (infet and surface roughness and immersed boundary conditions techniques ), ${ }^{18}$ or practical simulation issues related to grid sensitivity sudies. ${ }^{6}$ Physical simulaltons, in the context of the blind comparison, focused in the prediction of the speed-up, $S / S_{0}$, and the normalized increase of TKE, $\Delta \bar{k}$ (see Section 2 for detailed definitions of these parameters), at isoheight transects along defined wind directions and vertical transects at met mast locations (Figure 1). The physical simulations of the speed-up were considered by Bechmann et al. ${ }^{4}$ to achieve good agreement with full-scale results. A mean absolute speed-up error $\overline{\left|\epsilon_{\Delta S}\right|}=14 \%$ was reported as the average of the performance of the two physical simulations for cases 1 $\left(270^{\circ}\right)$ and $3\left(239^{\circ}\right)$, with the best physical model exhibiting a value $\overline{|\epsilon \Delta S|}=13.3 \%$. The reported mean absolute error in the determination of $\Delta \bar{k}$ was $\overline{|\epsilon \bar{k}|}=61.4 \%$, with the best physical model exhibiting a value $\overline{|\epsilon \bar{k}|}=59.4 \%$. The expressions for both performance indexes are described in Section 2 according to Bechmann et al. ${ }^{4}$

In this paper, a WT simulation of the flow on the Bolund island is described. Results are presented for two Reynolds numbers. Attention is paid to the detailed description of the inflow, the comparison with full-scale results and previous 
laboratory simulations, as well as to the spectral characterization of the flow. Emphasis is placed on the reproducibility and trustworthiness of results. For that purpose, redundancy measurements of PIV, two components hot-wire (2CHW) and three $3 \mathrm{CHW}$ anemometry were taken. The present work is organized as follows: in Section 2, the experimental setup is reported and the analysis applied to the measurements is formulated. The inflow conditions in the ACLA16 WT are presented in Section 3. Some insights on the flow topology on Bolund are introduced in Section 4. In Section 5, the new WT results are compared with full-scale data and previous WT and water channel measurements. Finally, in Section 6, conclusions are drawn.

\section{EXPERIMENTAL SET-UP AND MEASUREMENT ANALYSIS}

Two and three CHW and PIV tests were performed on a 1:115 scale model of the Bolund island, in the ACLA16 WT of IDR-UPM. The mock-up of the Bolund island was manufactured out of NECURON (NECUMER, Bohmte, Germany) by computer aided tooling, utilizing the same topography data file used by the numerical modellers during the blind test. ${ }^{19}$ The surface finishing of the mock-up is smooth. The front area of the mock-up is estimated to be less than $2 \%$ of the test section area, so blockage effects were expected to be minimized. No additional BL generators were used. This leads to a value of the ratio $\delta / h$ ( $\delta$ is the BL height) much smaller than the full-scale value. Some implications of this mismatch are discussed throughout the paper. A better matching of the ratio $\delta / h$ will be studied more carefully in on-going continuation experiments by employing BL generators such as the ones analysed in the study by Conan. ${ }^{13}$ The floor along the fetch was made out of plywood without any added roughness elements.

The ACLA16 WT is an open circuit, suction type, Eiffel WT, with a closed test chamber $(2.2 \mathrm{~m}$ wide and $2.2 \mathrm{~m}$ high). The convergent section is two-dimensional, with a length of $6 \mathrm{~m}$, leading into a straight test section of $18 \mathrm{~m}$, allowing the natural development of the BL. The WT is driven by 16 eight-bladed variable speed fans, with a nominal output of $10 \mathrm{~kW}$, capable of producing winds up to $30 \mathrm{~m} \mathrm{~s}^{-1}$. A dedicated traversing system was installed, allowing the displacement of a sensor in a volume of $2.0 \times 1.5 \times 1 \mathrm{~m}$ with a precision of $10 \mu \mathrm{m}$. The HW system is a DANTEC Streamware set-up with four channels. Constant temperature anemometers were used. DANTEC, two-wires probe (55P61) and three-wires probe (55P91) were used to simultaneously measure two or three components of flow velocity. Proper velocity and directional calibrations were applied to both probes. Temperature compensation was performed when required. In both cases, the procedures indicated in the study by Jørgensen ${ }^{20}$ were followed.

The HW probes were mounted on the appropriate supports, and they were automatically traversed to follow the required trajectories across the $\mathrm{BL}$ and above the model of the island. In order to facilitate the traversing of the probes close to the ground, the probe supports were mounted with their longitudinal axis, $x_{p}$, forming an angle $\Theta=15 \circ$ with the WT floor. The proper rotation matrix was applied to transform the velocities obtained in the probe reference system $\left[\tilde{u}_{p}, \tilde{v}_{p}, \tilde{w}_{p}\right]$ into the ones expressed in the Bolund reference system $[\tilde{u}, \tilde{v}, \tilde{w}]$ (Figure 1). The displacement of the probe on the island was programmed using the same topography data file used for the manufacturing of the mock-up. HW measurements were not taken in front of the escarpment because of the geometric constraints (collision of the probe support against the edge of the escarpment). The probes were sampled at $8 \mathrm{kHz}$ during $130 \mathrm{~s}$. A $3 \mathrm{kHz}$ low-pass hardware filtering was used when required. The $2 \mathrm{CHW}$ measurements were only taken for the determination of the inflow $\mathrm{BL}$ as an additional quality check.

The PIV system is a TSI Inc. set-up using a frame straddling approach. A pulsed Nd-YAG laser with a power of $200 \mathrm{~mJ}$ pulse ${ }^{-1}$, emitting a laser beam with a wavelength of $532 \mathrm{~nm}$ was used, along with a spherical and a cylindrical lens to illuminate the flow with a 1 to $2 \mathrm{~mm}$ width light sheet. Sebacate particles in the order of $1 \mu \mathrm{m}$ diameter were generated with a Laskin nozzle-based generator and were shed into the flow $10 \mathrm{~m}$ upstream of the model. The flow field images were captured with a $2048 \times 2048$ pixels charge-coupled device camera. The camera was positioned at a focus distance of roughly $0.8 \mathrm{~m}$ (Figure 1). The size of the image frame was about $0.20 \times 0.20 \mathrm{~m}$, and the capture rate was set at around four image pairs per second. Two rows, one on top of the other, with a total of 10 consecutive-overlapping frames each, were used to scan the whole length of the model. A pair of reference images with displacement markers were taken before each frame capture to allow the matching of the consecutive frames after processing. A set of 1000 image pairs were recorded for each frame. The camera focus and aperture were adjusted to have particle images about three to four pixels in size in order to minimize correlation noise. The appropriate time intervals between laser pulses were chosen, depending on the flow conditions, to obtain a displacement of the particle image about $25 \%$ of the final interrogation window size. A cross-correlation analysis of each pair of particle images was performed to resolve the velocity vector field. A multi-pass processing method with reducing interrogation window size with an initial interrogation window of $64 \times 64$ pixels with $50 \%$ overlap was used. The final interrogation window was set to $24 \times 24$ pixels, also with $50 \%$ overlap, to get the final vector field resolution. A Gaussian fit of the correlation peak was applied to ensure sub-pixel displacement accuracy and to minimize peak-locking effects. ${ }^{21}$ After masking the model and the outer borders of the image frames, the number of spurious vectors was limited to a maximum of $7 \%$, but usually, this number was much lower.

The analysis of the measurements closely followed the guidelines given in Bechmann $e t$ al. ${ }^{4}$ The measured instantaneous velocity vector, $\left[\tilde{u}_{1, n}, \tilde{u}_{2, n}, \tilde{u}_{3, n}\right]$, or, indistinctly, $\left[\tilde{u}_{n}, \tilde{v}_{n}, \tilde{w}_{n}\right]$, with $n=1, \ldots, N$, where $N$ is the number of time samples, 

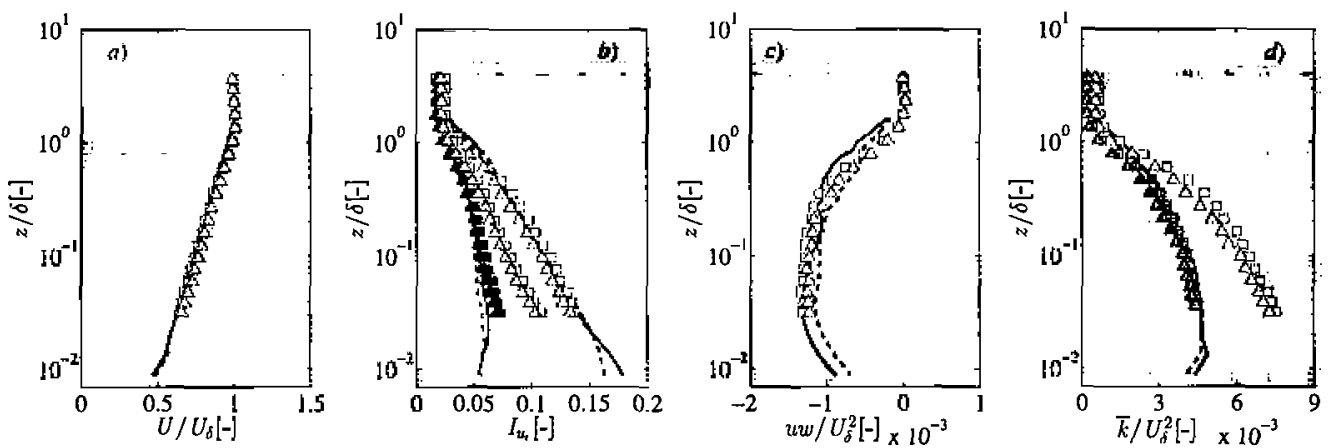

Figure 2. Basic statistics of the ACLA16 BL. Continuous lines, PIV for Re Ris; dashed lines, PIV for Re $e_{n 2}$; squares, $3 \mathrm{CHW}$ for Re $e_{11}$; triangles, $3 \mathrm{CHW}$ for $\mathrm{Re}_{h 2}$. In figure $b$ ): white symbols, $u$; yellow symbols, $v$; red symbols, $w$. In figure d), the green symbols represent the 'two-components' energy, $\bar{k}_{2} \mathrm{C}$ calculated from the $3 \mathrm{CHW}$ measurements.

is expressed by its components in the so-called Bolund reference system, $[x, y, z]$ in Figure 1. In this reference, the axis $x>0$ points to the east, the axis $y>0$ points to the north and the axis $z>0$ is the upward vertical. The $z$ coordinate represents the height above ground level. The absolute height or height above sea level is represented by $z_{\text {asl }}$. The mean velocity vectors are denoted by $\left[U_{1}, U_{2}, U_{3}\right]$ or $[U, V, W]$, and the instantaneous velocity fluctuation vectors are represented by $\left[u_{1, n}, u_{2, n}, u_{3, n}\right]$ or $\left[u_{n}, v_{n}, w_{n}\right]$. Therefore, the Reynolds decomposition of the velocity vector is $\tilde{u}_{i, n}=U_{i}+u_{i, n}$, where the mean velocity components are $U_{i}=N^{-1} \sum_{n=1}^{N} \tilde{u}_{i, n}$, being $i=1,2,3$; obviously, $N^{-1} \sum_{n=1}^{N} u_{i, n}=0$. The mean flow speed is $S=\left(U^{2}+V^{2}+W^{2}\right)^{1 / 2}$, where, in the case of the PIV measurements, $V=0$. Throughout this work, a 'two-components' mean flow speed, $S_{2 \mathrm{C}}=\left(U^{2}+W^{2}\right)^{1 / 2}$, is also used in some cases where PIV and 3CHW results are compared. The TKE is $\bar{k}=\left(\overline{u^{2}}+\overline{v^{2}}+\overline{w^{2}}\right) / 2$, where the overbar denotes averaging, which is $\bar{\bullet}=N^{-1} \sum_{n=1}^{N} \bullet$. From the PIV measurements, the total TKE cannot be calculated and a 'two-components' TKE, $\bar{k}_{2 \mathrm{C}}=0.5\left(\overline{u^{2}}+\overline{w^{2}}\right)$, is then obtained. The same parameter is also calculated from $3 \mathrm{CHW}$ measurements to allow the comparison with PIV results. The covariances of the velocity components, $\overline{u_{i} \bar{u}_{j}}$, are also determined so that the full Reynolds stress tensor is known. In the case of PIV, only the covariance $\overline{u w}$ is available. The turbulence intensities of the three velocity components are calculated as $I_{u_{i}}=\left(\overline{u_{i}^{2}}\right)^{0.5} U^{-1}$. The friction velocity $u_{*}$ and the aerodynamic roughness length $z_{0}$ are calculated from the fitting of the vertical profile of the mean flow speed, $U(z)$, to the well-known law of the wall, ${ }^{22}$ given by $U(z)=\kappa^{-1} u_{*} \ln \left(z / z_{0}\right)$, where $\kappa$ is the von Karman constant, here considered $\kappa=0.41$. In order to make the WT data comparable with the results reported in the studies by Berg et al. ${ }^{2}$ and Bechmann et al. ${ }^{4}$ the reference friction velocity $u_{* 05}$ (in the mentioned paper represented by $\left.u_{\star 0}\right)$ is calculated from the turbulent shear stress at $h_{0}(z=5 \mathrm{~m}$ in full-scale and $z=0.043 \mathrm{~m}$ in the WT) as $u_{* 05}=(-\bar{u} \bar{w})^{1 / 2}$. The reference height, $h_{0}$, is well within the surface layer (the region where the turbulent shear stress is almost constant) in the full-scale experiment. However, in the scaled experiment, the low height of the BL generated in the WT means that $h_{0}$ is out of this region. Although conceptually the value $\overline{u w}\left(h_{0}\right)$ should not be considered representative of the surface layer values of the WT BL, it is observed in Figure 2 (c) that the $\overline{u w}$ value at $h_{0}(z / \delta \approx 0.2)$ is quite similar to the surface layer value. The value of friction velocity obtained from the log profile is $25 \%$ higher than the value obtained from $\overline{u w}\left(h_{0}\right)$ (Table I); therefore, if $u_{*}$ is used as reference instead of $u_{* 05}$, the corresponding non-dimensional parameters would decrease accordingly.

The mean flow inclination angle is calculated from 3CHW measurements as $\Gamma=\arctan ^{-1}\left[W\left(U^{2}+V^{2}\right)^{-0.5}\right]$, whereas from PIV measurements, it is calculated as $\Gamma=\arctan ^{-1}\left(W U^{-1}\right)$. The mean flow direction angle is determined as $\Phi=\arctan ^{-1}\left(V U^{-1}\right)$. In Berg et al., ${ }^{2}$ the so-called deflection angle is presented as the difference between the wind direction measured at a certain location on the island and the wind direction measured at the reference position M0. Therefore, the full-scale deflection angle equals $-\Phi$.

Spectra and cospectra are calculated as $S_{i j}=\mathbb{R e}\left[\hat{u}_{i}(f) \hat{u}_{j}^{*}(f) T^{-1}\right]$, with $T$ being the duration of the time series, $\hat{u}_{i}(f)$ the Fourier transform of $u_{i}(t)$, the exponent * denotes complex conjugate and $f$ denotes frequency. The Welch's method is applied to obtain $S_{i j}$; therefore, each velocity fluctuation time series, with a length $N \gtrsim 2^{21}$ samples, is split into subsets of $2^{17}$ samples with $75 \%$ overlap. The spectral forms have been smoothed for presentation purposes using moving averaging. The quadrature spectra are not presented here. The one-sided representations of the spectral forms in terms of frequency are used throughout this work. Thus, the variance of the corresponding velocity component is $\overline{u_{i}^{2}}=\int_{0}^{\infty} S_{i i}(f) \mathrm{d} f$. In Berg et al., ${ }^{2}$ two-sided spectra and cospectra are used; therefore, the spectral functions presented here are twice the ones shown in the mentioned reference. The Kaimal's one-sided spectral forms as presented in 
Table I. Main characteristics of the undisturbed inflow $\mathrm{BL}$ for $\mathrm{Re}_{h 1}=4.15 \times 10^{4}$ and

\begin{tabular}{|c|c|c|c|c|c|c|c|}
\hline & \multirow[b]{2}{*}{ Full scale } & \multicolumn{3}{|c|}{$\operatorname{Re}_{h 1}$} & \multicolumn{3}{|c|}{$\mathrm{Re}_{h 2}$} \\
\hline & & $2 \mathrm{CHW}$ & $3 \mathrm{CHW}$ & PIV & $2 \mathrm{CHW}$ & $3 \mathrm{CHW}$ & PIV \\
\hline$\delta[\mathrm{m}]$ & - & 0.240 & 0.220 & - & 0.230 & 0.210 & - \\
\hline$u_{*}\left[\mathrm{~m} \mathrm{~s}^{-1}\right]$ & - & 0.314 & 0.293 & 0.306 & 0.596 & 0.572 & 0.565 \\
\hline$u_{* 05}\left[\mathrm{~m} \mathrm{~s}^{-1}\right]$ & 0.47 & 0.270 & 0.267 & 0.247 & 0.497 & 0.520 & 0.484 \\
\hline$z_{0} \times 10^{5}[\mathrm{ml}$ & 60 & 1.04 & 1.08 & 1.02 & 0.52 & 0.54 & 0.59 \\
\hline$\theta \times 10^{3}[\mathrm{~m}]$ & - & 20.0 & 20.3 & - & 18.4 & 18.1 & - \\
\hline$h^{-1} \delta$ & - & 2.343 & 2.148 & - & 2.245 & 2.050 & - \\
\hline$\left.I_{u}\right|_{h_{0}} \times 10^{2}$ & 12.0 & 9.3 & 10.0 & 9.5 & 8.6 & 9.2 & 9.4 \\
\hline $\mid I_{h_{0}} \times 10^{2}$ & 8.0 & - & 7.1 & - & - & 6.6 & $=$ \\
\hline$\left.I_{w}\right|_{h_{0}} \times 10^{2}$ & 5.0 & 5.1 & 5.5 & 4.9 & 4.9 & 5.1 & 4.7 \\
\hline$I_{u} I_{h} \times 10^{2}$ & - & 7.3 & 7.5 & 7.3 & 6.4 & $6 ; 8$ & 7.4 \\
\hline$\left.I_{v}\right|_{h} \times 10^{2}$ & - & - & 5.4 & - & - & 5.0 & - \\
\hline$\left.I_{w}\right|_{h} \times 10^{2}$ & - & 4.1 & 4.3 & 4.3 & 3.9 & 4.0 & 3.9 \\
\hline$h^{-1} L_{u}^{x}$ & - & - & 3 & - & - & 3.5 & - \\
\hline$U_{\delta} \theta v^{-1} \times 10^{-4}$ & - & 1.04 & 1.00 & - & 1.92 & 1.80 & - \\
\hline$u_{*} z_{0} v^{-1}$ & 18 & 0.219 & 0.212 & 0.208 & 0.206 & 0.205 & 0.226 \\
\hline $\mathrm{Je}=h z_{0}^{-1} \times 10^{-4}$ & 1.96 & 0.98 & 0.94 & 1.00 & 1.97 & 1.90 & 1.70 \\
\hline$S_{m} \times 10^{4}$ & 1.041 & 0.98 & 0.93 & - & 1.31 & 1.14 & - \\
\hline
\end{tabular}

The lengths $\delta$ and $z_{0}$ for the WT simulations are shown in WT scale. The declared interval for the fullscale Reynolds number is $4.25 \times 10^{6} \leq \operatorname{Re}_{h} \leq 10.2 \times 10^{6}$. A reference value $\delta=0.22 \mathrm{~m}$ is selected hereafter. $\theta$ is the momentum thickness.

the study by Kaimal and Finnigan ${ }^{23}$ are used here as references. Also, as indicated in Berg et al., ${ }^{2}$ the spectral functions are calculated from the velocity components projected in the so-called local horizontal wind reference system, which is a reference system resulting after rotating the Bolund reference system the mean flow direction angle, $\Phi$, around the $z$ axis.

The inflow integral length scales of the velocity fluctuations in the longitudinal direction, $L_{u_{i}}^{x}, i=1,2,3$, are calculated from the corresponding time constants, $\tau_{i}$, and the mean velocity component, $U$, using the Taylor's hypothesis of frozen turbulence, as $L_{u_{i}}^{x}=U \tau_{i}$, where $\tau_{i}$ is estimated from the approximation $\overline{u_{i}(t) u_{i}\left(t+\tau_{i}\right)}=\mathrm{e}^{-1}$.

From the previous measurements, the speed-up is calculated as $S(x, y, z) S_{0}^{-1}(z)$, where $S(x, y, z)$ stands for the mean flow speed at the analysed location $[x, y, z]$, and $S_{0}(z)$ is the mean speed at the same height above ground level, $z$, but in a convenient upstream reference position (M0 in the full-scale experiment). The normalized increase of TKE, $\Delta \bar{k}$, is calculated as $\Delta \bar{k}=\left[\bar{k}(x, y, z)-\bar{k}_{05}\right] S_{0}^{-2}(z)$, where $k_{05}=k_{0}\left(z=h_{0}\right)$ is the TKE in the upstream reference position, measured at $h_{0}$ (again following Bechmann et al. ${ }^{4}$ ). Since the $h_{0}$ reference point is well out the surface layer in the WT, the used reference value $k_{05}$ is $25 \%$ lower than the typical surface layer value (Figure 2 ). Therefore, the values presented here for the normalized increase of TKE are slightly higher than those obtained using the typical surface layer TKE value as reference.

\section{ANALYSIS OF THE INFLOW CONDITIONS IN THE WT}

The WT tests were run at two Reynolds numbers, $\operatorname{Re}_{h 1}=4.15 \times 10^{4}$ and $\mathrm{Re}_{h 2}=8.21 \times 10^{4}$. For surface mounted obstacles, as in this case, where $h<\delta$, the obstacle is well immersed in the turbulent $\mathrm{BL}$, and values $\operatorname{Re}_{h}>(2-3) \times 10^{4}$ have been usually considered sufficiently high to neglect Reynolds number effects. In the wording of Lim et al., ${ }^{24}$ this assumption has formed the basis of most WT scaled simulations for the past half century. In the mentioned reference, it was concluded that this is true for bluff bodies, where mean flow quantities are concerned (velocity and pressure) and no strongly concentrated, or relatively steady vortex regions exist in the flow field (i.e. conical vortex on roofs oriented at $45^{\circ}$ to the incoming flow). Where such vortex structures exist, their viscous cores topologies present a strong dependency on $\mathrm{Re}_{h}$ for values much larger than the referred threshold, transmitting this dependency on $\mathrm{Re}_{h}$ to the topology of the mean flow in those regions nearby the viscous vortex cores (these steady structures are not expected on Bolund since the vortex motions on the island are expected to be less prevalent). Regarding fluctuation statistics (mainly for the surface pressure variance), the dependence on $\mathrm{Re}_{h}$ has been proven beyond the previous limit, even when such strongly concentrated or relatively steady vortex regions are not present in the flow. As Lim et al. established, identifying genuine Reynolds number effects when comparing results of WT simulations and full-scale tests can be highly difficult because of the known effects of changes in upstream turbulence and length scale, basically quantified by different values of the parameters $I_{u}$ and $L_{u}^{x} h^{-1}$ determined at height $h$. The mismatch in the inflow spectral energy content for high frequencies between full-scale and WT BLs can also be responsible of differences in the prediction of detachment topologies. In this case, a modification 
of Melbourne's 'small scale' spectral density parameter (in the wording of Lim et al. ${ }^{24}$ ), $S_{m}$, is reported to quantify the high-frequency energy content of the inflow.

No thermal stratification was reproduced in the WT ( $\mathrm{Fr} \rightarrow \infty$ ) so the Froude number similarity was relaxed, which is accurate given the value of the full-scale Monin-Obhukov length. The Rossby number similarity was also relaxed given the size of the Bolund island. The Prandtl number similarity was fulfilled since the fluid was air, and since the flow was incompressible, the Eckert number similarity was also satisfied.

Particle image velocimetry, $2 \mathrm{CHW}$ and $3 \mathrm{CHW}$ redundancy (but not simultaneous) measurements of the inflow BL were performed. The WT reference inflow conditions were determined from independent tests with the empty test section. The HW measurements were obtained in a vertical transect at an equivalent full-scale position $x=-80 \mathrm{~m}, y=0 \mathrm{~m}$. The PIV measurements were obtained from sets of 1000 pairs of images centred in the same position. The reference measurements were taken at the two Reynolds numbers $\operatorname{Re}_{h 1}$ and $\mathrm{Re}_{h 2}$ in all cases. The main parameters of the simulated WT-BL are indicated in Table I to illustrate the degree of agreement between the different techniques, which is generally good. Some dispersion is found in the values of the aerodynamic roughness length $z_{0}$ (and in the parameters derived from this value such as the Jensen number, Je) which is expected due because of the known uncertainties of the indirect method used here in its determination. ${ }^{25}$ ) The WT results for the three turbulence intensities at $z=h_{0}$ are representative of the full-scale values.

From the $z_{0}$ values reported in Table I, a roughness Reynolds number $u_{*} z_{0} v^{-1} \approx 0.21$ was adopted. Thus, the inflow should be considered transitionally rough, taking into account the lower limit of the fully rough regime, $u_{*} z_{0} v^{-1}=5$, and the upper limit of the fully smooth one, $u_{*} z_{0} v^{-1}=0.2$, as indicated in the study by Bowen, ${ }^{16}$ although in the study by Snyder and Castro, ${ }^{26}$ the lower limit for the fully rough regime is reduced down to $u_{*} z_{0} v^{-1}=1$ and even down to $u_{*} z_{0} v^{-1}=0.5$ for certain roughness geometries. Similar conditions were described for the WT simulations of Askervein in the study by Teunissen $e$ al al., ${ }^{11}$ where values $u_{*} z_{0} v^{-1}=0.16,0.54$ and 1.7 , respectively, were declared, or in the study by Røkenes and Krogstad, ${ }^{27}$ where a value $u_{*} z_{0} v^{-1}=0.13$, well within the smooth regime, was reported. As in the mentioned experiments, the present case should be understood as the simplest possible reference case. Some possible effects of the reported low value for $u_{*} z_{0} \nu^{-1}$ are discussed in the succeeding text.

As mentioned before, a modification of the Melbourne's parameter is determined as $S_{m}=f_{c} S_{11}\left(f_{c}\right) U_{c}^{-2}$, with $f_{c}=n_{c} U_{c} h_{c}^{-1}$. In the study by Lim et al. ${ }^{24} n_{c}=10, U_{c}=U_{h}$ and $h_{c}=h$. However, in the present work, full-scale spectra are only available at $h_{0}$ and for a maximum frequency $f_{\max }=4.79 U_{h_{0}} h_{0}^{-1}$. Thus, here, $S_{m}$ is calculated using $h_{c}=h_{0}, U_{c}=U_{h_{0}}=U\left(z=h_{0}\right)$ and $n_{c}=4.79$. The resulting value of this parameter at full-scale is $S_{m}=1.041 \times 10^{-4}$ similar to the WT values (Table I).

The reference vertical profiles for the mean wind speed $U(z)$, the turbulence intensity of the three velocity components, $I_{u_{i}}(z), i=1,2,3$, the turbulent shear stress, $\overline{u w}(z)$, and the TKE, $\bar{k}(z)$, have been represented in Figure 2 . The mean wind speed is presented scaled with its value at the BL height $U_{\delta}$, whereas both the turbulent shear stress and the TKE are presented scaled with $U_{\delta}^{2}$. The detailed analysis on scaling considerations taking into account variations of the Reynolds number based in the momentum thickness, $U_{\delta} \theta \nu^{-1}$, is out of the scope of the present work (for details, see Bakken $e t$ al. ${ }^{28}$ ). Here, it is only mentioned that the outer scaling (using $U_{\delta}$ and $\delta$ ) of the presented magnitudes shows certain independency on the Reynolds number. The agreement between PIV and $3 \mathrm{CHW}$ measurements is quite acceptable.

The horizontal statistical homogeneity of the velocity field with regard to the mean longitudinal velocity component, $U$, and its standard deviation, $\left(\overline{u^{2}}\right)^{1 / 2}$, has been verified from $3 \mathrm{CHW}$ measurements at two equivalent full-scale heights, $z=2 \mathrm{~m}$ and $z=5 \mathrm{~m}$. The variation of both parameters along the longitudinal coordinate, $x$, at $y=0$, is presented in Figure 3(a). The streamwise transects roughly span one island length, and they are centred in the test section. The results for the lateral transect are shown in Figure 3(b). These transects are taken roughly at $2 h$ upstream of the island escarpment and roughly span 1.5 times its width. The acceleration parameter $K=\nu U^{-2} \mathrm{~d} U / \mathrm{d} x$ was in all cases $K<1.6 \times 10^{-6}$, and
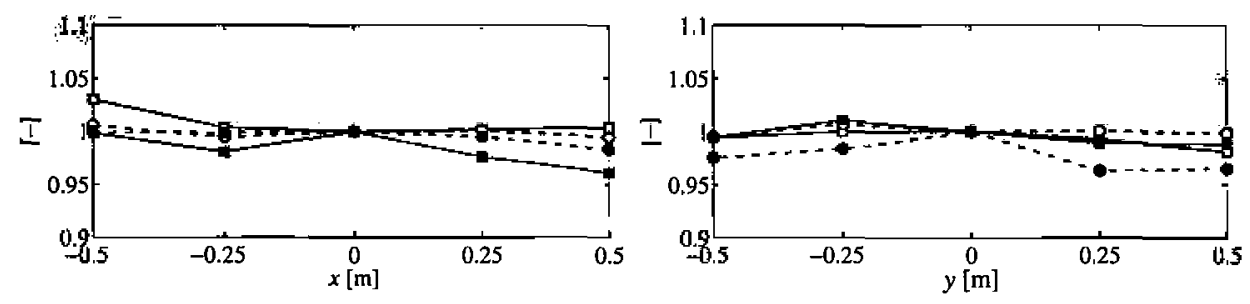

Figure 3. a) Longitudinal ( $x$-wise) and b) lateral ( $y$-wise) variation of the mean of the longitudinal component of the wind velocity, $U$ (closed symbols) and its standard deviation, $\left(\overline{u^{2}}\right)^{1 / 2}$ (open symbols) at $z=2 \mathrm{~m}$ (squares) and $z=5 \mathrm{~m}$ (circles). The results are presented scaled with the value at $x=0$ and $y=0$, respectively. 
therefore, according to DeGraaff and Eaton, ${ }^{29}$ no significant influence of the pressure gradient on the mean speed profile is expected.

The one-sided spectra in the inflow BL are presented in Figure 4 for $\mathrm{Re}_{h 1}$. The spectra are shown for six heights covering the whole BL thickness. The Kaimal models for the three spectra and the ESDU model for the longitudinal one ${ }^{30}$ are also shown. The expected behaviour in the low frequency range $\left(S_{i i} \sim f^{0}\right)$ and in the inertial sub-range $\left(s_{i i} \sim f^{-5 / 3}\right)$ are clearly discernible at every height. The Kaimal model especially matches the spectra of the three velocity components at non-dimensional heights $z / \delta=0.1$ and 0.2 . The agreement is maintained for the longitudinal and vertical components at $z / \delta=0.3$; it is not so good for $z / \delta=0.05$ and $z / \delta=0.5$, and it is poor for $z / \delta=1$, which is expected since the Kaimal's functions were developed to match the spectral forms in the surface layer. It is remarked that the matching with the ESDU form for $S_{11}$ is poor in general.

To facilitate the comparison of the main characteristics of the WT-BL with the full-scale ABL, the vertical profile of the wind speed scaled with $u_{* 05}$, and the TKE scaled with $u_{* 05}^{2}$, as they were presented in the study by Bechmann et al., are shown in Figure 5. The full-scale values of $\bar{k} u_{* 05}^{-2}$ at $z=5 \mathrm{~m}$ and $z=12 \mathrm{~m}$ are quite similar $(\approx 5.4)$; in fact, in the study by Bechmann et al. ${ }^{4}$ a constant value $\bar{k} u_{* 05}^{-2}=5.8$ for numerical modelling purposes was proposed. It is evident that the WT vertical profiles $\bar{k}(z) u_{* 05}^{-2}$ and $\bar{k}_{2 \mathrm{C}}(z) u_{* 05}^{-2}$ clearly decreased with height. This mismatch of the full-scale TKE
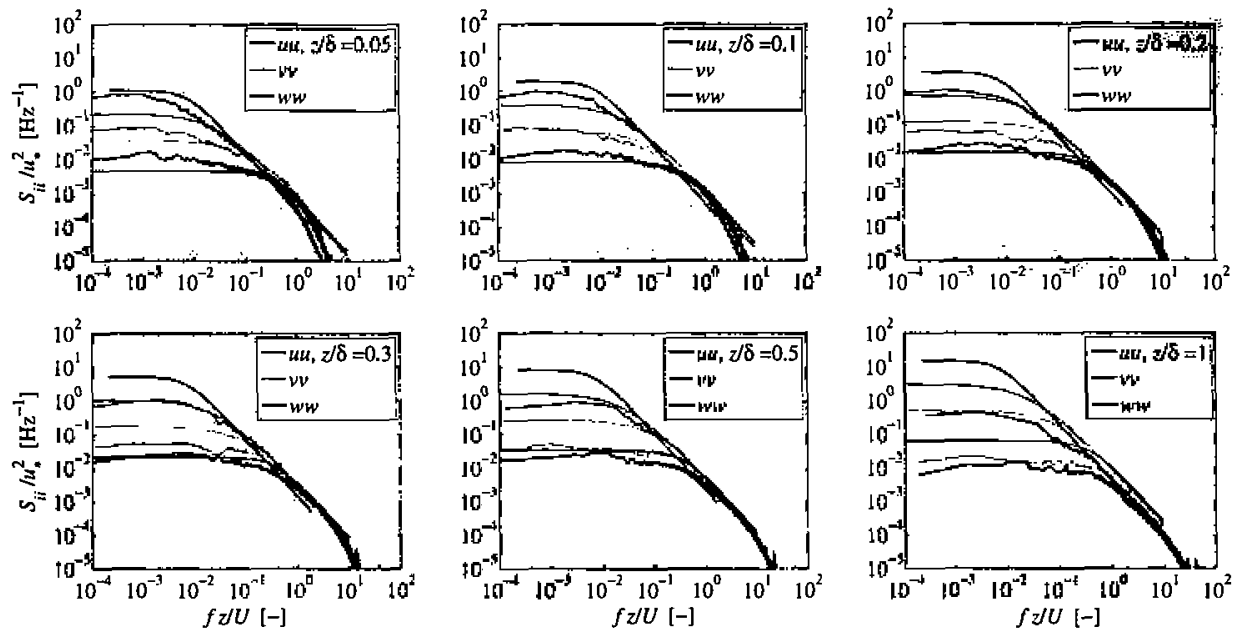

Figure 4. $3 \mathrm{CHW}$ measurements (thick lines) of the one-sided spectra in the ACLA16 BL, corresponding to the longitudinal, lateral and vertical velocity fluctuations, $S_{i i}$, scaled with the squared value of the friction velocity. $U_{*}$. The spectra are presented for different heights indicated in the figures as the corresponding ratio $z / \delta$. The thin lines represent the Kaimal model for the corresponding spectrum, ${ }^{23}$ whereas the black lines correspond to the ESDU model for $S_{11}$. ${ }^{30}$ The WT results were obtained for Re $\theta_{h 1}$.
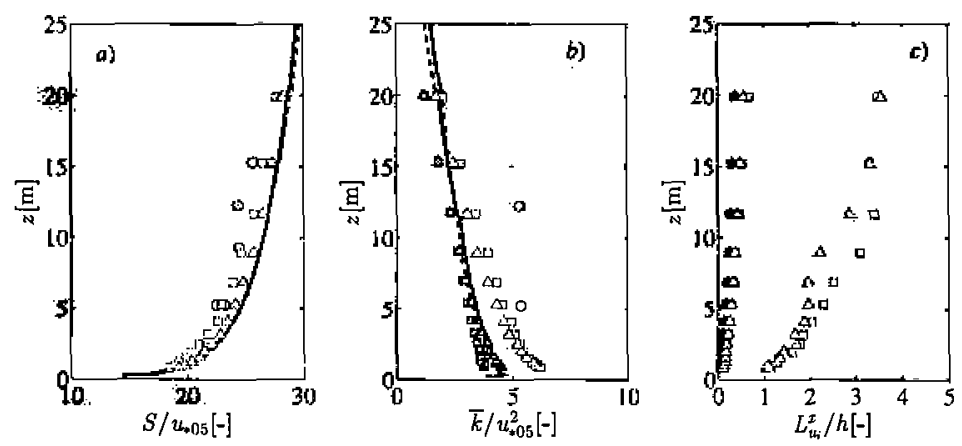

Figure 5. Inflow profiles of the following: a) wind speed, $S$, scaled with $\left.u_{* 05}, b\right)$ TKE, $\bar{k}$, scaled with $u_{* 05}^{2}$ and $c$ ) integral length scales of the velocity components along the longitudinal direction, $L_{u,}^{x}$, scaled with $h$. Symbols and lines are coded as in Figure 2 . Yellow circles represent the full-scale data from the study by Bechmann et al. ${ }^{4}$ 
profile is directly related to the earlier commented low value of the modelled BL height compared with the full-scale value. The real island is fully immersed in the surface layer, where the TKE is fairly constant with height, whereas in the scaled experiment, the TKE varies about $50 \%$ along the mock-up height. A better matching of the TKE profile is planned in future WT experiments.

The vertical profiles of the longitudinal integral length scales of the three velocity components, $L_{\boldsymbol{u}_{i}}^{x}, i=1,2,3$, are reported in Figure $5(\mathrm{c})$. The values presented are scaled with the height of the island, $h$. The values $L_{u_{i}}^{x} h^{-1}$ for the full-scale test have not been declared anywhere, to the authors' knowledge, but considering the length of the sea fetch, a full-scale value $L_{u_{i}}^{x} h^{-1}>10$ is likely, therefore much bigger than in the WT simulation (Figure 5(c) and Table I).

\section{INSIGHTS ON THE FLOW TOPOLOGY ON BOLUND}

Some characteristics of the flow on Bolund that are especially challenging for modellers are outlined. The escarpment facing the westerly wind directions provokes a strong deceleration of the flow upstream of the island, for $z<h$, see Figure 6(a), together with a high increase of the surface pressure, as reported in Yeow et al. ${ }^{15}$ This deceleration was measured in full-scale ${ }^{2}$ and was generally well reproduced by numerical models ${ }^{4}$ and WT tests. ${ }^{13,14}$ A slight increment of TKE was measured in full-scale met masts, i.e. M7 for the $270^{\circ}$ wind direction. This increment was reproduced by some of the numerical models in the blind comparison, and it is evident in Figure $6(b)$. This increment of TKE is associated to the presence of intermittent re-circulation regions where velocity components $\tilde{u}<0$ are likely to occur. This likelihood of upstream re-circulation was analysed in the study by Yeow et al..$^{14}$ and is reproduced in Figure 6(c), where the values $\log _{10}[p(\tilde{u}<0)]$ are shown, where $p()$ means probability. Although a re-circulation pattern is not identified in the mean velocity field, such as in the case of a two-dimensional facing forward step (2D-FFS; see the study by Yeow $e$ a $a .^{31}$ and references therein), there are regions upstream close to the escarpment where $p(\tilde{u}<0)>10 \%$. The authors have previously documented the existence of instantaneous vortex structures by PIV images in this region. ${ }^{14}$

Flow detachment takes place at the escarpment edge. Intermittent re-circulation patterns on top of the island, downstream of the edge, have been identified from full-scale measurements of ultrasonic anemometers at low heights for $239^{\circ}$ wind direction ${ }^{2,32}$ and have been documented from Lidar measurements for $270^{\circ}$ wind direction. ${ }^{17}$ Indirect 'symptoms' of these detachment re-circulation patterns are the high values of normalized increase of TKE (Figures 6(b), 7 and 15). The authors of the present work have also provided direct measurements of such intermittent re-circulation patterns for the $270^{\circ}$ wind direction case from PIV analysis, ${ }^{14}$ where some instantaneous velocity fields corresponding to the re-circulation-nore-circulation states were shown. This detachment pattern is identified in Figure $6(\mathrm{c})$, where regions with $p(\tilde{u}<0)>0$ appear on the top on the island. There are enough evidences to establish that the detachment for line $B$ on the Bolund island is an intermittent process with relatively low frequency of occurrence and a mean re-circulation bubble (such as the one developed on a 2D-FFS) does not take place.

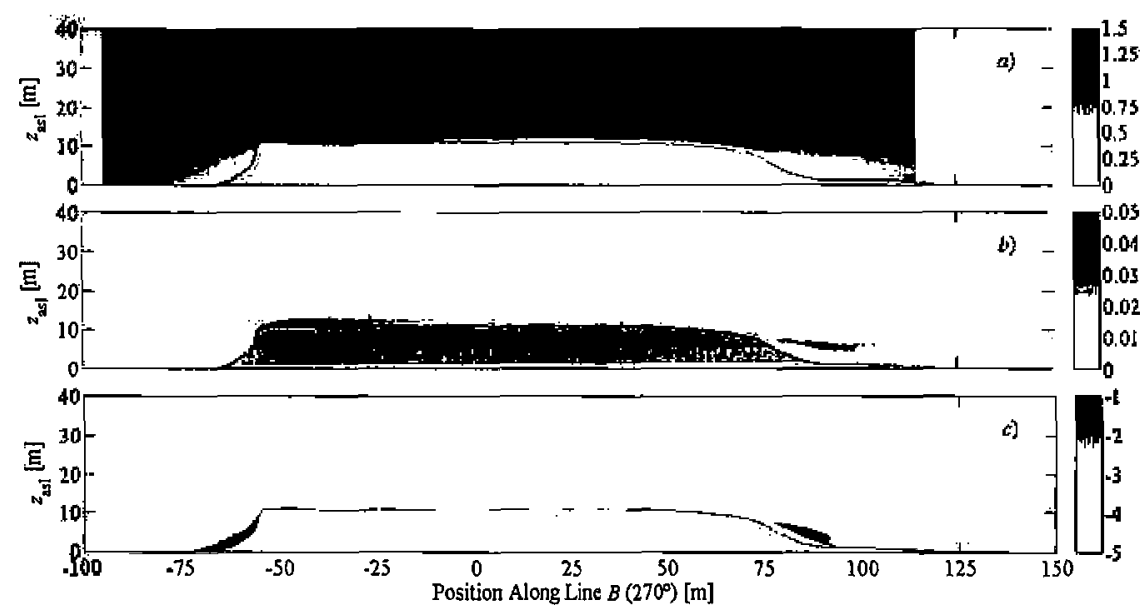

Figure 6. a) Speed-up, $\left.S / S_{0}, b\right)$ normalized increase of TKE, $\Delta \bar{k}_{2 c}$ and $c$ ) probability of occurrence of instantaneous negative values of the $\tilde{u}$ component of the velocity vector, represented as $\log _{10}[p(\tilde{u}<0)]$. Results for $R_{h 2}$. The white region directly on the lee side corresponds to a loss of field view by the PIV camera because of the topography. 


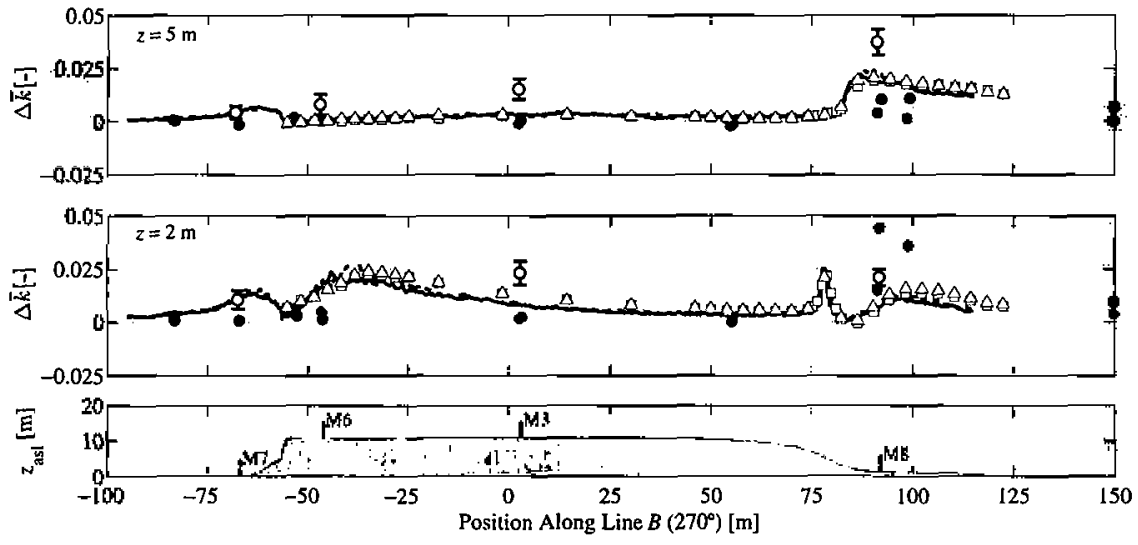

Figure 7. Normalized increase of TKE, $\Delta \bar{k}$. Continuous lines, 2 CPIV for Re $e_{h 1}$; dashed lines, 2 CPIV for $R_{h 2}$; squares, $3 C H W$ for $R_{h 1}$; triangles, $3 \mathrm{CHW}$ for $\mathrm{Re}_{h 2}$. Full-scale results (yellow dots with uncertainty bars), previous water channel results (red dots) and previous WT results (blue dots) from the study by Bechmann. ${ }^{32}$ The full-scale value at $2 \mathrm{~m}$ in $\mathrm{M6}$ does not appear in the figure and its value is $\Delta \vec{k}=0.125$.

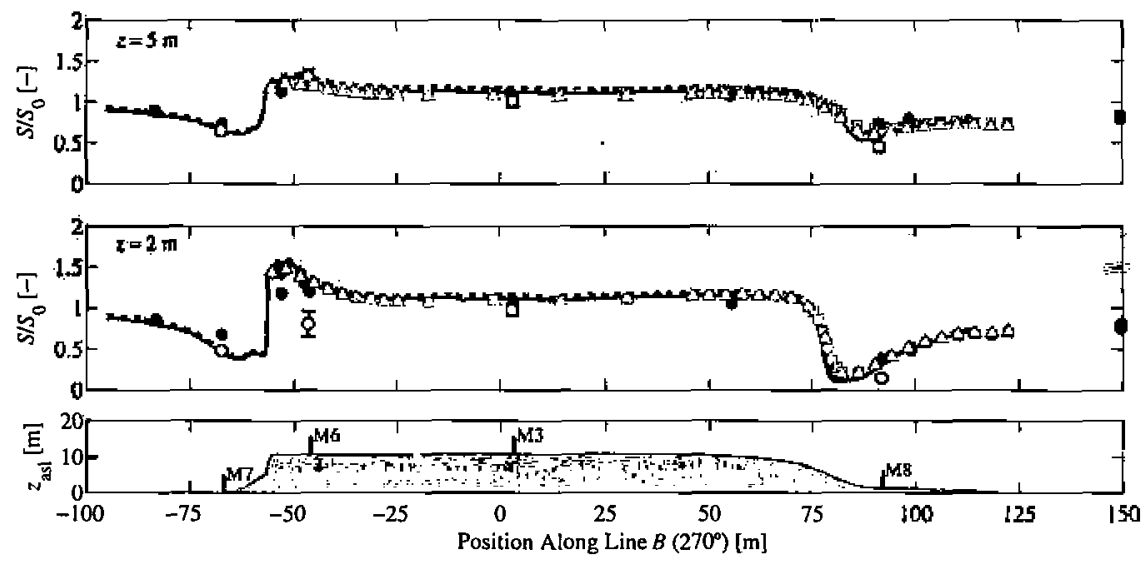

Figure 8. Speed-up, $S / S_{0}$. Coding as in Figure 7.

Also, indirect evidences on the existence of such pattern are the highly negative values of the mean pressure coefficient and the high values of its standard deviation on the surface of the island, previously documented by the authors. ${ }^{15} \mathrm{~A}$ highly turbulent shear layer developed above and downstream of the intermittent re-circulation region and extending till $x \sim-25 \mathrm{~m}$ is present, which is a characteristic that is also observed on the re-circulation bubble on an FFS. In previous works, the authors have estimated the most likely position of the re-attachment area on top of the island after the escarpment edge. This analysis was performed for different flow directions by applying well-known criteria on the basis of statistical properties of the pressure field on the island surface. These criteria were derived from the analysis of the mean re-circulation patterns on 2D-FFSs. Although in the Bolund case the geometry differs from a 2D-FFS and the probability of instantaneous re-circulation patterns is not high enough as to lead to a re-circulation bubble in the mean velocity field, the applicability of these statistical criteria were proven in the study by Yeow et al..${ }^{15}$ also for this case.

After the intermittent re-circulation area, the so-called relaxation region follows (using Bradshaw wording ${ }^{33}$ ), where the velocity field evolves towards horizontally homogeneous conditions. For $x \gtrsim 75 \mathrm{~m}$, a wake develops in the lee side of the island. This region is characterized by a large speed reduction (see full-scale results in Figures 8 and 14) and a high normalized increase of TKE (see full-scale results for M8 in Figure 15). Considering Figure 6(b),(c), the wake region seems to be characterized by the presence of a shear layer developing above and downstream of the intermittent re-circulation area. 


\section{COMPARISON OF WT RESULTS FOR TRANSECT B}

In this section, the results for the transect $B$, according to the format proposed in the study by Bechmann $e t a l^{4}$, are presented for the speed-up, $S / S_{0}$, the normalized increase of TKE, $\Delta \vec{k}$, the inclination angle, $\Gamma$, and, finally, the deflection angle, $-\Phi$. All these parameters have been calculated as indicated in Section 2 . The evolution of the mentioned parameters at $z=2 \mathrm{~m}$ and $z=5 \mathrm{~m}$ on the island has been obtained from PIV and 3CHW measurements, for the two considered Reynolds numbers, $\operatorname{Re}_{h 1}$ and $\mathrm{Re}_{h 2}$. Full-scale results and results from the simulations in WT and water channel reported in the context of the blind comparison experiment are included (when available) for comparison purposes.

In Figure 8, the speed-up $S / S_{0}$ is shown. The behaviour in the upstream location (M7) is generally well predicted. A mismatch in the prediction of the speed-up in the positions just after the escarpment at $z=2 \mathrm{~m}$ (corresponding to M6 position in full scale) is evident. It is observed that all scaled simulations seriously over-predict the speed-up in this region, which, as it has been described in Section 4, presents a highly complex flow topology exhibiting intermittent re-circulation patterns and high TKE. This mismatch does not exist at $z=5 \mathrm{~m}$, which is expected, since this location is out of the intermittent re-circulation area as can be seen in Figure 6(b), where the extension of the shear layer does not reach $z=5 \mathrm{~m}$.

An overestimation of the speed-up is predicted in the relaxation region (M3). This is in agreement with the physical models reported in the blind comparison, and it has also been found in some recent new WT experiments on the Bolund Island for quite different inflow conditions. ${ }^{13}$ In the lee side of the island, the agreement between WT results is high, though they exhibit certain overestimation of the speed-up compared with full-scale measurements. No significant differences between both Reynolds number cases are found.

It is reminded that in the present case, $u_{*} z_{0} \nu^{-1} \simeq 0.21$, meaning that the inflow is most likely in the transitionally rough regime. The roughness of the model is also low, so re-laminarisation in the relaxation region after the re-circulation area cannot be rejected. This fact was identified in the study by Bowen ${ }^{16}$ as a cause of the over-prediction of the speed in the wake region of Askervein (the lee sides of Askervein and Bolund are somehow similar). In fact, the WT simulations described in the study by Conan ${ }^{13}$ were most likely performed in the fully rough regime (BL developed on Lego floor and terraced model), and they exhibited speed-up values at $z=5 \mathrm{~m}$ for M8, very well fitting the full-scale values.

A large under-prediction of $\Delta \bar{k}$ just after the escarpment (M6) for $z=2 \mathrm{~m}$ exists (the full-scale value at $2 \mathrm{~m}$ in M6 is out of the $y$-axis limits, its value being $\Delta \bar{k}=0.125$ ). The WT experiments predict a value for $\Delta \bar{k}$ one order of magnitude lower than the full-scale one. The reduced values of the parameters $\operatorname{Re}_{h}$ and $L_{u}^{x} h^{-1}$ in the WT could partially justify this result. However, the mismatch is considered too large as to be produced only by this scale effect. For instance, in the study by Lim et al., ${ }^{24}$ a similar experiment has been conducted with a cube in the free atmosphere $\left(\operatorname{Re}_{h}=3 \times 10^{6}\right)$ and in a WT $\left(\operatorname{Re}_{h}=2 \times 10^{4}\right)$, and underestimations of $50 \%$ in the parameter $\overline{u^{2}} U_{h}^{-2}$ have been reported for the WT simulation. The lack of fidelity in the modelization of the escarpment edge is proposed as a likely, partial explanation of the underestimation of $\Delta \bar{k}$ in the present case. In Figure 9, it is evident that the mock-up does not reproduce the sharpness of the real edge. With regard to this fact, in the study by Røkenes and Krogstad, ${ }^{27}$ the characteristics of the flow in the WT on a straight slope

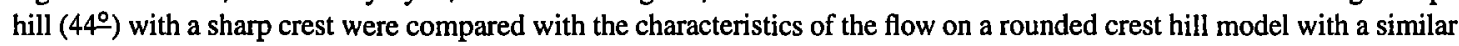
slope. This comparison revealed large discrepancies in both the speed-up and the turbulence increase. Thus, in the sharp edge case, mean decelerations, which is $S / S_{0}<1$, at low heights after the edge were reported (as in the Bolund prototype), whereas, in the rounded edge case, values $S / S_{0}>1$ were observed everywhere on top of the hill (as in the Bolund model). On the other hand, the increase of the parameter $\overline{u^{2}} U_{0}^{-2}$ for the sharp edge case was one order of magnitude higher than
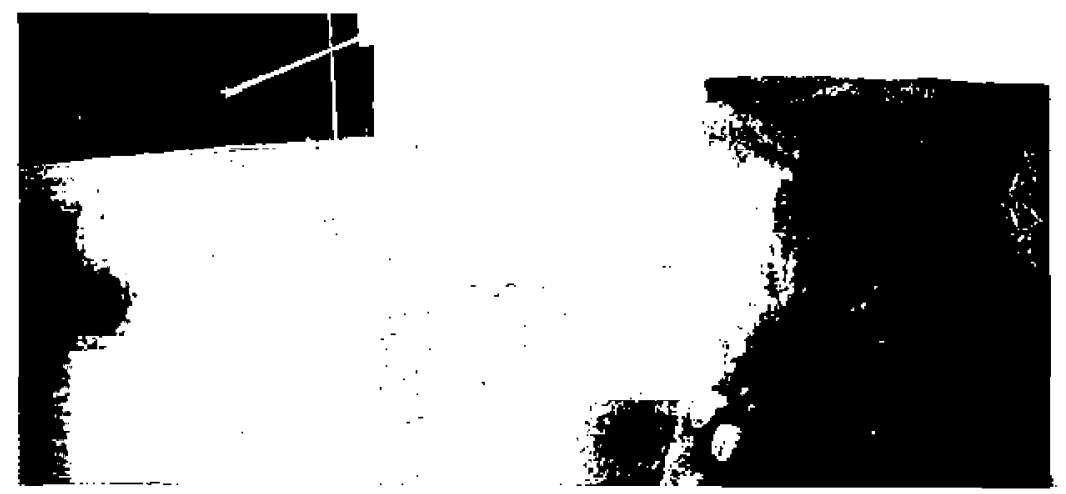

Figure 9. Escarpment edge of the Bolund island. Left: 1:115 tested model roughly viewed from the south-west direction. Right: real topography roughly viewed from the south direction. 
the corresponding increment for the rounded edge model, which is in agreement with the discrepancies in $\Delta \bar{k}$ found in this work between full-scale and WT measurements.

The inclination angle, $\Gamma$, is generally well reproduced (Figure 10). However, a high deviation is found for $\Gamma$ in M6 location at $z=2 \mathrm{~m}$, where the PIV and $3 \mathrm{CHW}$ predict similar values $\Gamma \sim 0 \circ$ but the ultrasonic anemometer measures a value $\Gamma \sim-12.5$. . The other relevant characteristic is the large difference observed in the lee side of the island at $z=2 \mathrm{~m}$ between the PIV and $3 \mathrm{CHW}$ estimations of $\Gamma$. The PIV results agree quite well with the full-scale measurements whereas the $3 \mathrm{CHW}$ results do not. In this region, the probability of having instantaneous flow angles out of the acceptance cone of the $3 \mathrm{CHW}$ probe is high. The determination of the inclination angle is especially sensitive to this effect, more than $S / S_{0}$ or $\Delta \bar{k}$ where as shown before, exhibits coherence for both techniques. The $3 \mathrm{CHW}$ probe limitations in situations where large instantaneous flow angles relative to the probe axis are present is further analysed in Section 5.1 .

The deflection angle, $-\Phi$, is presented in Figure 11. The results compare well with the full-scale data at M6 and M3 (within the uncertainty of the angular calibration of the probe ${ }^{34}$ ). At M8, the 'out of acceptance cone' effect, as in the case of the inclination angle, introduced a large bias in the determination of the deflection angle.

In order to quantify the bias of the simulated speed-up, the speed-up error $\epsilon_{\Delta S}=100\left(\Delta S_{W T}-\Delta S_{F S}\right)$ has been calculated, and to quantify the bias in the determination of the normalized increase of TKE, the parameter $\epsilon \bar{k}$ given by

$$
\epsilon_{\Delta \bar{k}}=100\left[\left(\frac{\sqrt{\bar{k}}}{S_{0}}-\frac{\sqrt{\bar{k}_{05}}}{S_{0}}\right)_{W T}-\left(\frac{\sqrt{\bar{k}}}{S_{0}}-\frac{\sqrt{\bar{k}_{05}}}{S_{0}}\right)_{F S}\right]\left(\left.\frac{\sqrt{\bar{k}_{05}}}{S_{0}}\right|_{F S}\right)^{-1}
$$
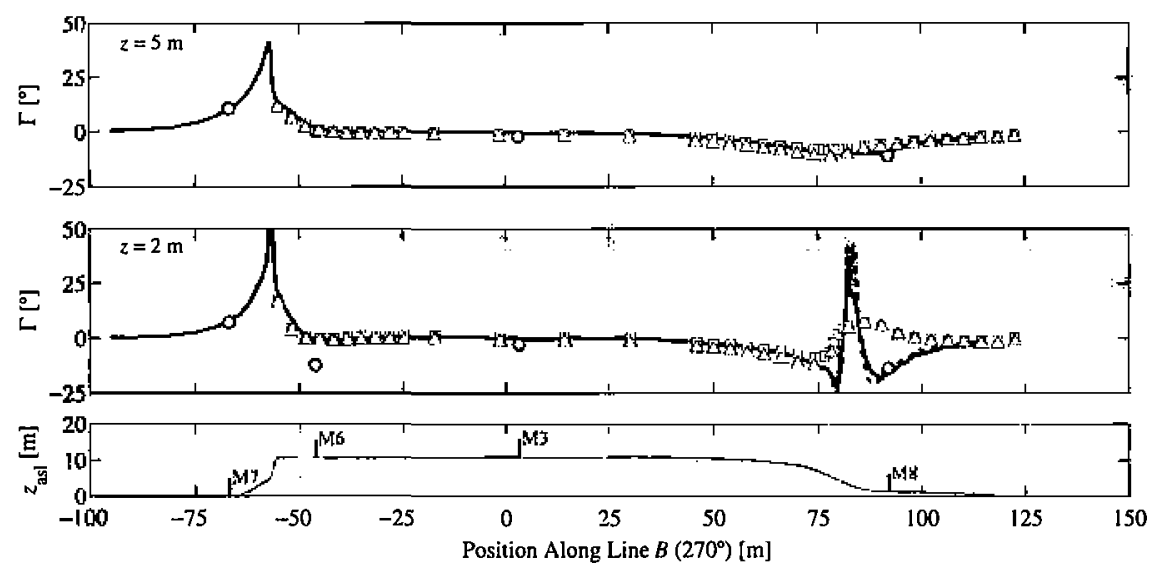

Figure 10. Inclination angle, $\Gamma$. Coding as in Figure 7.

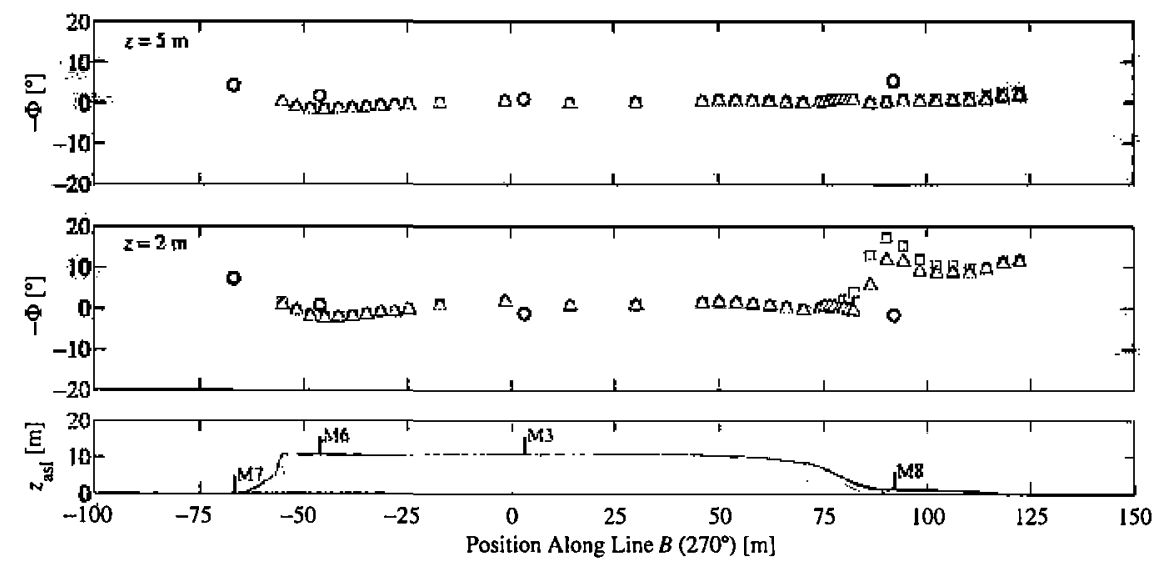

Figure 11. Deflection angle, $-\Phi$. Coding as in Figure 7. 
has been obtained. The mean absolute values for $\epsilon_{\Delta S}$ and $\epsilon_{\Delta \bar{k}}$ are presented in Table II. The bias corresponding to $3 \mathrm{CHW}$ measurements are calculated only for locations M6, M3 and M8 since HW measurements at M7 location were not taken because of geometric constraints. The bias from PIV measurements included also M7, which in general presented a low error. Therefore, to allow the comparison between $3 \mathrm{CHW}$ and PIV bias, the mean absolute errors from PIV results without including $\mathrm{M} 7$ are reported in brackets. With regard to the mean absolute value of $\epsilon_{\Delta S}$, PIV and 3CHW show typical maximum differences of two percentage points. The bias from PIV measurements are in agreement with the values reported in the study by Bechmann et al. ${ }^{4}$ There, PIV measurements clearly outperform the 3CHW measurements in relation to $\epsilon_{\Delta \bar{k}}$. The mean absolute values of this parameter obtained from PIV results are 15 percentage points lower than the values reported in the study by Bechmann $e t$ al. ${ }^{4}$ for the highest performing model. Increasing the Reynolds number from $\operatorname{Re}_{h 1}$ to $\operatorname{Re}_{h 2}$ does not mean a significant improvement in the mean absolute value of $\epsilon \Delta S$ in the case of the $3 \mathrm{CHW}$ (from $20.67 \%$ down to $19.40 \%$ ). The bias goes slightly higher in the case of PIV (from $13.87 \%$ to $16.19 \%$ ) when the Reynolds number is increased, which is not a natural trend. However, in the case of $\epsilon_{\Delta \bar{k}}$, a higher Reynolds number leads to a coherent reduction in $\overline{|\epsilon \bar{k}|}$ of roughly seven percentage points for both techniques.

\subsection{Validation of HW measurements in highly inclined-turbulent flow regions}

In those regions where the instantaneous flow velocity formed a large angle with the probe axis, $\mathrm{HW}$ measurements are likely to be largely biassed since the instantaneous flow velocity is likely to be out of the acceptance cone of the probe $\left(\alpha_{\max }=35^{\circ}\right.$ for $55 \mathrm{P} 91$ probe. $\left.{ }^{3534}\right)$ In order to identify these regions, the probability of having instantaneous velocity vectors forming angles with the probe axis larger than $\alpha_{\max }$ has been calculated from the instantaneous PIV velocity fields and presented in Figure 12. Since the PIV only provides the components $\tilde{u}$ and $\tilde{w}$ of the instantaneous velocity vector in $x z$ planes, the analysis is only performed in the $x z$ plane, and the calculated probability is $p\left(\tilde{\alpha}_{x z} \geq \alpha_{\max }\right)$, being $\tilde{\alpha}_{x z}=\arccos \left\{\mid[\tilde{u}, 0, \tilde{w}] \cdot[\cos \Theta, 0, \sin \Theta]\left\{\left(\tilde{u}^{2}+\tilde{w}^{2}\right)^{-1 / 2}\right\}\right.$, where $\Theta$ is the pitch angle of the HW probe (Figure 1), whereas a more rigorous verification should take into account the true instantaneous angle, $\tilde{\alpha}=\arccos \left\{|[\tilde{u}, \tilde{v}, \tilde{w}] \cdot[\cos \Theta, 0, \sin \Theta]|\left(\tilde{u}^{2}+\tilde{v}^{2}+\tilde{w}^{2}\right)^{-1 / 2}\right\}$.

The problematic regions (i.e. where $p\left(\tilde{\alpha}_{x z} \geq \alpha_{\max }\right) \geq 0.05$ ) are located just in front of the escarpment, on the top of the island just after the escarpment and on the lee side of the island. These regions roughly coincide with the recirculation areas (those regions where $p(\tilde{u}<0)>0$ ) shown in Figure $6(\mathrm{c})$. However, it is remarked that the regions where $p\left(\tilde{\alpha}_{x z} \geq \alpha_{\max }\right)>0$ are significantly larger than those regions where some likelihood of re-circulation exists, meaning that the instantaneous re-circulation states are only a part of the problematic situations with regard to the 'out of acceptance angle' condition. When the instantaneous velocity vector is out of the acceptance cone of the probe, the calibration function of the sensor that relates the voltages measured by the probe wires and the velocity components is badly conditioned. This produces biassed calibrated velocity components that can even be complex values in extreme cases. The percentage of

Table II. Mean values of the absolute errors in the determination of the speed-up, $\overline{\left|\epsilon_{\Delta s}\right|}$ and in the normalized increase of TKE, $\overline{\left|\epsilon_{\Delta \bar{k}}\right|}$, in different met masts locations and heights.

\begin{tabular}{lcccc}
\hline & & & \multicolumn{2}{c}{$\bar{j} \epsilon_{\Delta \bar{x}} \mid$} \\
\hline & $3 C H W$ & $2 C P I V$ & $3 C H W$ & $2 C P I V$ \\
$R e_{h 1}$ & 20.67 & $13.87(17.96)$ & 69.38 & $47.91(60.53)$ \\
$\operatorname{Re}_{h 2}$ & 19.40 & $16.19(21.05)$ & 64.34 & $43.72(53.69)$ \\
\hline
\end{tabular}

The mean absolute values of the bias for M6, M3 and M8 from PIV measurements are indicated in brackets.

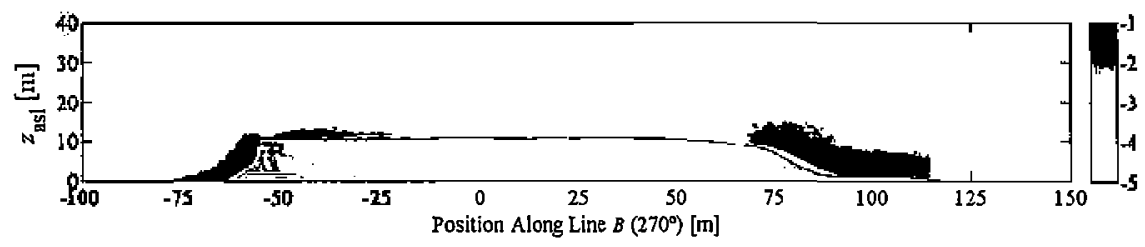

Figure 12. Probability of occurrence of an instantaneous velocity vector out of the vertical acceptance angle of the HW probe. The colour scale corresponds to $\log _{10}\left[p\left(\tilde{\alpha}_{x z} \geq \alpha_{\text {max }}\right)\right]$. Result for Re $e_{h 2}$. The white region directly on the lee side corresponds to a loss of field view by the PIV camera because of the topography. 


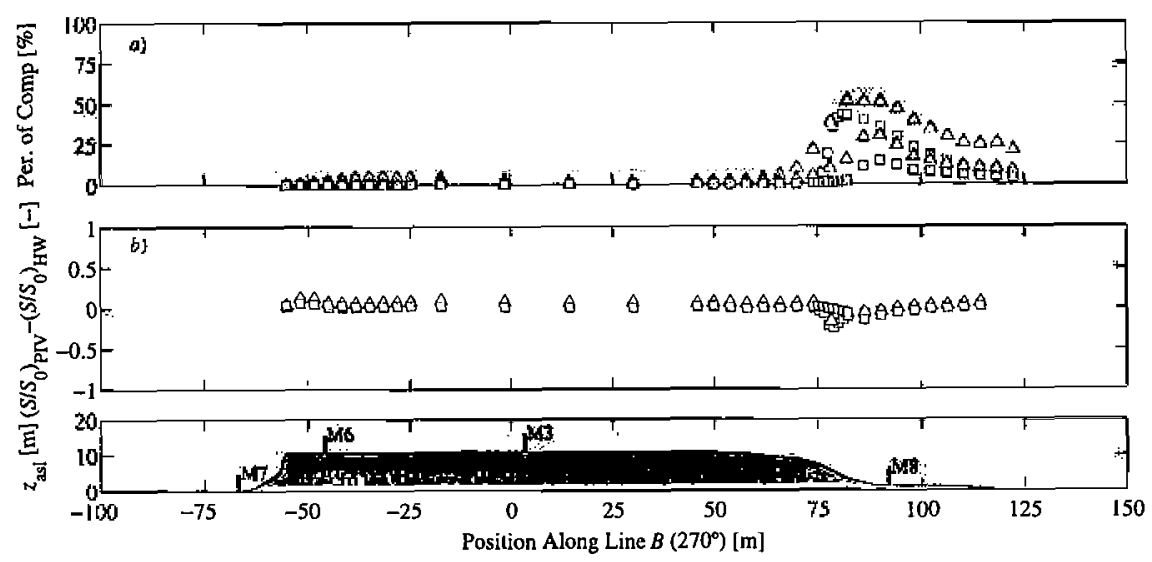

Figure 13. a) Percentage of complex valued calibrated velocities. b) Difference from speed-up, $S_{2 c} S_{0}^{-1}$, predicted from $3 \mathrm{CHW}$ and PIV measurements. White symbols, $z=2 \mathrm{~m}$; grey symbols, $z=5 \mathrm{~m}$; squares, $\operatorname{Re}_{h 1}$; triangles, Re $e_{22}$.
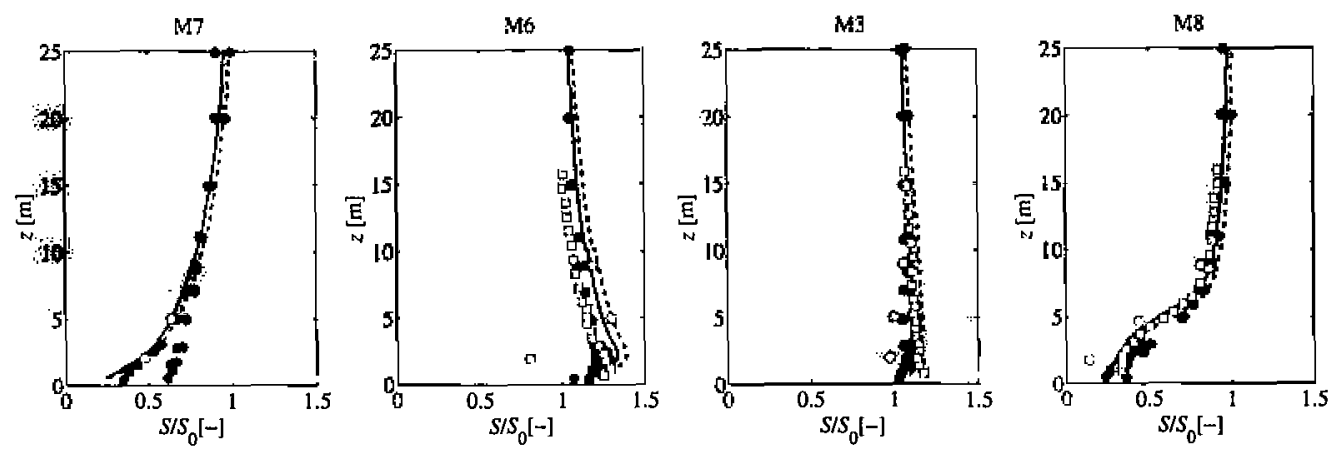

Figure 14. Speed-up, $S / S_{0}$, at met masts $M 7, M 6, M 3$ and $M 8$. Continuous lines, 2CPIV for Re $e_{h 1}$; dashed lines, $2 C P I V$ for Re ${ }_{m 2}$; squares, $3 \mathrm{CHW}$ for $\mathrm{Re}_{h 1}$. Full-scale results (yellow dots), previous water channel results (red dots) and previous $W T$ results (blue dots) from the study by Bechmann. ${ }^{32}$

complex valued calibrated velocity components for each time series are shown in Figure 13. It can be observed that the higher occurrence of complex values takes part where $p\left(\bar{\alpha}_{x z} \geq \alpha_{\max }\right)$ is significantly high.

\subsection{Met mast analysis.}

The vertical profiles of $S / S_{0}$ and $\Delta \bar{k}$ at M7, M6, M3 and M8 locations are analysed. Only PIV results are presented in the upstream region of the island (M7). PIV measurements were taken for both Reynolds numbers, $\operatorname{Re}_{h 1}$ and $\mathrm{Re}_{h 2}$, whereas $3 \mathrm{CHW}$ ones were taken only for $\mathrm{Re}_{h 1}$ because of time restrictions.

Figure 14 reveals the significant agreement between the WT and full-scale values of the speed-up. This agreement is especially good in front of the escarpment (M7), in the re-circulation region (M6) for the higher positions and in the wake (M8). In M6, it is evident in the large bias in the prediction of $S / S_{0}$ at $z=2 \mathrm{~m}$ (location fully immersed in the re-circulation area). The results for $\mathrm{M} 3$ confirm the already commented overestimation of $S / S_{0}$ in the middle of the relaxation region. This behaviour is common for all the scaled simulations.

The WT measurements of $\Delta \bar{k}$ presented in Figure 15 reveal a good agreement with full-scale results, predicting exactly the values at M7 and the trends in M6, M3 and M8. The PIV results for $\Delta \bar{k}_{2 C}$ exhibit values of the same order of the full-scale ones at $z=2 \mathrm{~m}$ in M6 but at a much lower height ( $z=0.75 \mathrm{~m}$ in full-scale). An expected negative normalized increase of TKE is observed at larger heights for all met masts, specially in the relaxation region (M3), which is agreement with the findings reported in the study by Røkenes and Krogstad ${ }^{27}$ for the flow above embankments with roughly similar geometry. 

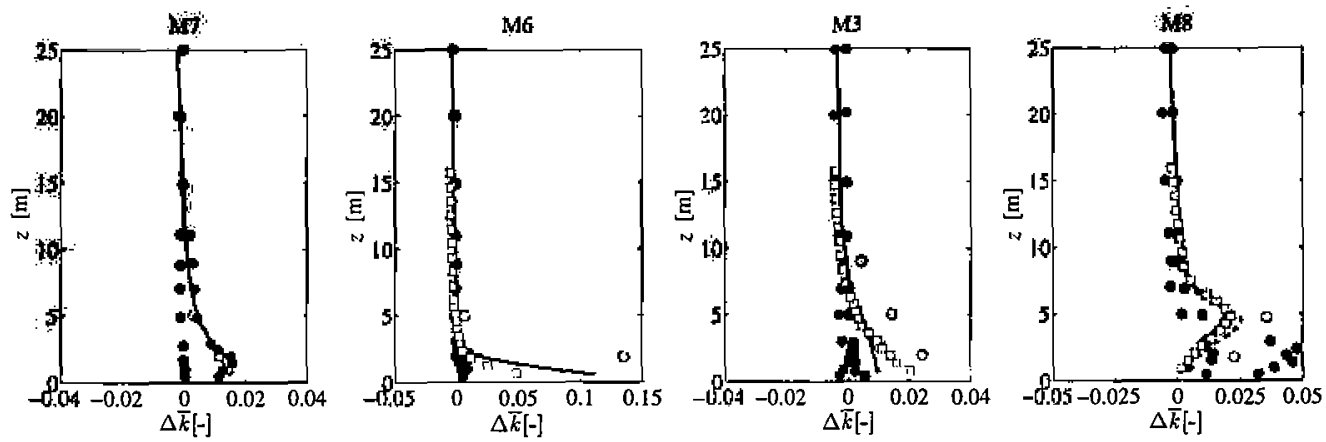

Figure 15. Normalized increase of TKE, $\Delta \dot{\bar{k}}$, at met masts $M 7, M 6, M 3$ and $M 8$. Continuous lines, 2CPIV for Rent; dashed lines, $2 \mathrm{CPIV}$ for Re $\mathrm{e}_{22}$; squares, $3 \mathrm{CHW}$ for Re $\mathrm{e}_{h 1}$. Full-scale results (yellow dots), previous water channel results (red dots) and previous WT results (blue dots) from the study of Bechmann. ${ }^{32}$
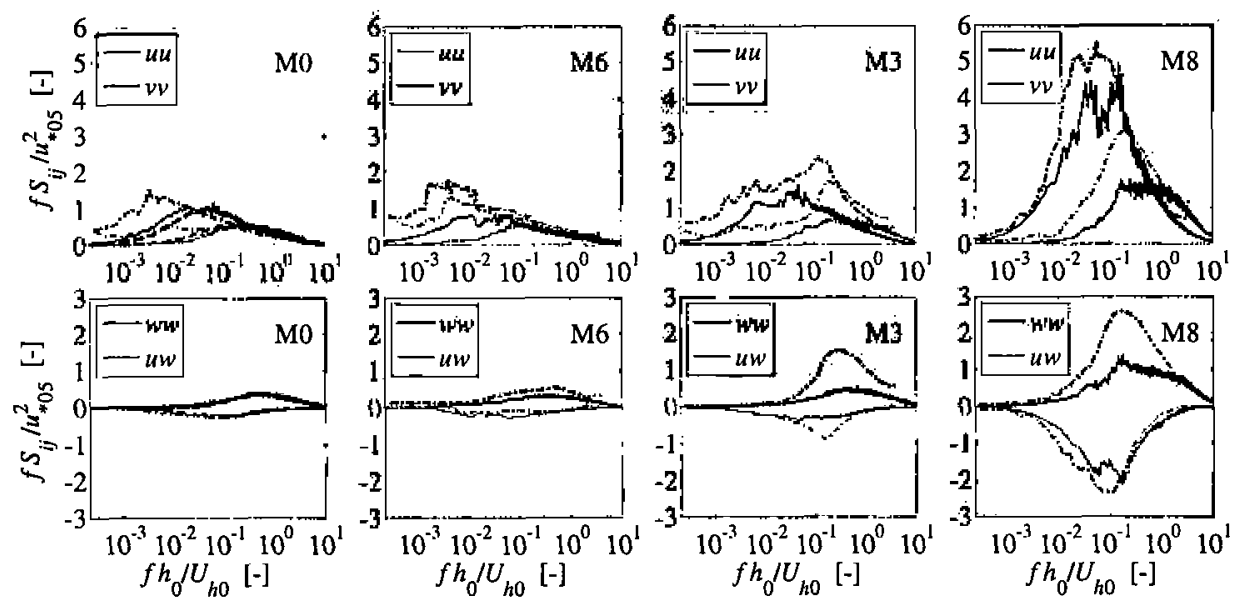

Figure 16. Normalized spectra, $S_{i i}$, and cospectrum, $S_{13}$, at $z=h_{0}$ at the met mast locations M0, M6, M3 and M8. The units used for non-dimensionalization are $h_{0}, U\left(h_{0}\right)=U_{m}$ and $U_{* 05}$. The dotted-dashed lines correspond to full-scale results, the solid lines to WT results and the dashed lines for $\mathrm{MO}$ to the Kaimal model. ${ }^{23}$

\subsection{Spectral analysis}

The normalized spectra and $u w$ cospectrum at $z=h_{0}$ for the reference position (equivalent to M0) and in the met mast positions M6, M3 and M8 have been determined from the velocity time series measured by the 3CHW and compared with the full-scale measurements. The results are presented in Figure 16 as $f S_{i j} u_{* 05}^{-2}$ (Section 2) and in terms of the nondimensional frequency $f h_{0} U_{h_{0}}^{-1}$, as proposed in the study by Berg et al. ${ }^{2}$ The inflow spectra at M0 are compared with the Kaimal spectral functions. It is evident in the good agreement between the Kaimal models and the WT results. In all cases, the energy content of the full-scale spectra and $u w$ cospectrum for the low frequency range is higher than the WT counterparts as expected. This is especially evident in the case of $S_{11}$ and $S_{22}$, where the maxima of the normalized spectra (associated with the integral length scale of the corresponding velocity component in the longitudinal direction) are clearly shifted to the lower frequencies (larger scales). The full-scale spectrum $S_{22}$ presents a more broadly distributed energy content compared with the WT simulation. After the maximum of the normalized spectra and cospectrum, which is for the higher frequencies, both the Kaimal and WT spectral functions exhibit higher levels of normalized energy compared with the full-scale ones.

The WT results predict qualitatively well the full-scale results for the locations analysed on the island. The underprediction of the low frequency energy levels in M6 for the $u u$ and $v v$ spectra is evident; however, the behaviour of the spectra for high frequencies is very well reproduced. Surprisingly, at M3, in the middle of the plateau, the under-prediction of the energy levels extends to the whole frequency range. The WT spectral functions in M8 (the location at $z=h_{0}$ is fully immersed in the shear layer of the island wake above the lee side re-circulation region) reproduced quite satisfactorily the 
full-scale results. The good prediction of the $u w$ cospectrum deserves attention. With this regard, it can be observed that the frequency distribution of the largely negative Reynolds shear stress is quite well captured in the WT at M8 location.

\section{CONCLUSIONS}

An attempt at reproducing the Bolund experiment at two Reynolds numbers in a WT has been presented. The WT measurements have been compared with a satisfying degree of agreement with the full-scale results of the Bolund blind comparison, which prove that WT modelling can provide a significant amount of accurate information on the flow topology over complex terrains.

The vertical scans of the velocity from the PIV measurements confirmed that the steep escarpment is always going to be the critical point in terms of flow field modification induced by the topography. Although a re-circulation bubble has not been detected in the mean velocity field, an intermittent re-circulation area with low probability of occurrence has been predicted from the existence of instantaneous negative horizontal velocity components in velocity fields determined from PIV measurements. A shear layer forming on the re-circulation region and evolving downstream over the plateau of the Bolund island has been visualized from the normalized increase of TKE. The analysis of this shear layer is crucial for studies of wind turbine siting as this region of the flow is characterized by a high value of TKE, which is a driver factor of fatigue loading of wind turbines. Putting this into the perspective of wind turbine siting on similar locations, the extension of the shear layer forming over an escarpment of 10 times the height of Bolund will be in the range of heights of interest where wind turbine loading is concerned.

At the critical location M6, close to the edge of the escarpment, a mismatch has clearly been seen at $2 \mathrm{~m}$ height (this location is within the thickness of the shear layer) for the speed-up and the normalized increase of TKE. The TKE has been under-predicted to a lesser extend at M3 and at M8 in the wake of the Bolund island where the flow is highly turbulent corresponding to the existence of an intermittent re-circulation area and a shear layer on it. The typical ideas about the cause for this mismatch, such as the differences in Reynolds number $\mathrm{Re}_{h}$ and turbulence intensity $I_{u}$ and the mismatch of the large and small scales of turbulence quantified by different values of $L_{u}^{x} h^{-1}$ and Melbourne's parameter, respectively, have been discussed along with modelling fidelity issues such as the influence of surface roughness or the accuracy in the reproduction of the sharpness of the escarpment edge. A detailed study of the regions of highly complex flow topology has addressed the issue of the limited angular response of the three components HW probes from the probability plot of velocity vectors exceeding their acceptance angle.

The results from the WT simulations are comparable with the Bolund experiment results in terms of the mean absolute error quantification for the speed-up, and they are significantly better in the case of the mean absolute error related to the prediction of normalized increase of TKE, with an improvement of 15 percentage points, from $59.4 \%$ declared for the highest performing physical model in the blind comparison down to $43.72 \%$ for the PIV measurements at the highest Reynolds number in the present work.

The spectral analysis has provided physically sound insights on how the topography modifies the energy content of the velocity fluctuations and its frequency distribution. In most of the cases, the predictions agreed qualitatively and also quantitatively with the full-scale results, providing valid information about spectra and cospectra.

It must be considered that the ratio of the BL height to the island height in the WT is much smaller than in the full-scale case. This mismatch leads to the following: (i) a poor reproduction of the TKE inflow profile along the island height, which can have certain influence (that should be further analysed) in the perturbation of the flow induced by the island simulated in the WT and (ii) the need to take into account that the reference values for the friction velocity and inflow TKE taken at $z=5 \mathrm{~m}$ in the WT are not representative of the scaled surface layer as they are in the full-scale case. A better matching of the inflow conditions is planned for near future tests.

\section{ACKNOWLEDGEMENTS}

This work was carried out as a part of the activities supported by the European Commission in the framework of the Marie Curie Action ITN-238576 WAUDIT. The authors wish to thank the technical staff in IDR-UPM for their contribution. The authors also wish to thank VKI for the valuable collaboration and RIS $\emptyset$-DTU for the full-scale data from the Bolund experiment.

\section{REFERENCES}

1. Taylor PA, Teunissen HW. Askervein hill project: overview and background data. Boundary-Layer Meteorology 1985; 39: 15-39.

2. Berg J, Mann J, Bechmann A, Courtney MS, Jørgensen HE. The Bolund experiment, part i: flow over a steep, three-dimensional hill. Boundary-Layer Meteorology 2011; 141(2): 219-243. DOI:10.1007/s10546-011-9636-y. 
3. Walmsley $\Omega$, Taylor PA. Boundary-layer Flow over topography: impacts of the Askervein study. Boundary-Layer Meteorology 1996; 78: 291-320.

4. Bechmann A, Sørensen NN, Berg J, Mann J, Rethore PE. The Bolund experiment, part ii: blind comparison of microscale flow models. Boundary-Layer Meteorology 2011; 141(2): 219-243. DOI:10.1007/s10546-01 1-9637-x.

5. Castro FA, Palma JMLM, Lopes AS. Simulation of the Askervein flow. Part 1: Reynolds averaged Navier-Stokes equations (k-epsilon turbulence model). Boundary-Layer Meteorology 2003; 107(3): 501-530. DOI: $10.1023 / \mathrm{A}: 1022818327584$.

6. Prospathopoulos JM, Politis ES, Chaviaropoulos PK. Application of a 3D Rans solver on the complex hill of Bolund and assessment of the wind flow predictions. Journal of Wind Engineering and Industrial Aerodynamics 2012; 107: 149-159. DOI:10.1016/j.jweia.2012.04.011.

7. Silva Lopez A, Palma JMLM, Castro FA. Simulation of the Askervein flow. Part 2: large-eddy simulations. Boundary-Layer Meteorology 2007; 125(1): 85-108. DOI:10.1007/s10546-007-9195-4.

8. Chow FK, Street RL. Evaluation of turbulence closure models for large-eddy simulation over complex terrain: flow over Askervein hill. Journal of Applied Meteorology and Climatology 2009; 48(5): 1050-1065. DOI:10.1175/2008JAMC1862.1.

9. Bechmann A, Sørensen NN. Hybrid RANS/LES method for wind flow over complex terrain. Wind Energy 2010; 13: 36-50. DOI:10.1002/we.346.

10. Mann J. The spectral velocity tensor in moderately complex terrain. Joumal of Wind Engineering and Industrial Aerodynamics 2000; 88: 153-169. DOI:10.1016/S0167-6105(00)00046-5.

11. Teunissen HW, Shokr ME, Bowen AJ, Wood CJ, Green DWR. The Askervein hill project - wind-tunnel simulations at 3 length scales. Boundary-Layer Meteorology 1987; 40(1-2): 1-29. DOI:10.1007/BF00140067.

12. Stock DE, Bowen AJ. Wind tunnel measurements of the turbulence structure of wind flow over Askervein hill, 10th American Meteorological Society Symposium on turbulence and diffusion, American Meteorological Society, Boston, 1992; J73-J75.

13. Conan B. Wind resource assessment in complex terrain by wind tunnel modelling. PhD. Report. Technical Report, Université d'Orléans, 2012.

14. Yeow TS, Cuerva A, Conan B, Pérez J. Wind tunnel analysis of the detachment bubble on Bolund island, The science of making torque from wind, Oldenburg, October 9-11, 2012.

15. Yeow TS, Cuerva A, Pérez J. Pressure Measurements of the detachment bubble on the Bolund island, European wind energy association, Copenhagen, April 16-19, 2012.

16. Bowen AJ. Modelling of strong wind flows over complex terrain at small geometric scales. Journal of Wind Engineering and Industrial Aerodynamics 2003; 91: 1859-1871. DOI:10.1016/S0167-6105(00)00046-5.

17. Mann J, Angelou N, Mikkelsen T, Hansen KH, Cavar D, Berg J. Laser scanning of a recirculation zone on the Bolund escarpment, The science of making torque from wind, Oldenburg, October 9-11, 2012.

18. Jafari S, Chokani N, Abhari RS. An immersed boundary method for simulation of wind flow over complex terrain. Journal of Solar Energy Engineering-Transactions of the ASME 2012; 134(1): 011006-011006-12. DOI: $10.1115 / 1.4004899$.

19. Bechmann A, Johansen J, Sørensen NN. The Bolund experiment-design of measurement campaign using CFD. Technical Report Ris $\phi-R-1623(E N)$, Risø, 2007.

20. Jørgensen FE. How To measure turbulence with hot-wire anemometers-a practical guide. Technical Report, DANTEC Dynamics, 2002.

21. Raffel M, Willert C, Wereley S, Kompenhans J. Particle Image Velocimetry. A Practical Guide. 2ed. Springer-Verlag: Berlin, 2007.

22. Pope SB. Turbulent Flows. Cambridge University Press: Cambridge, 2000.

23. Kaimal JC, Finnigan JJ. Atmospheric Boundary Layer Flows. Their Structure and Measurement. Oxford University Press: Oxford, 1994.

24. Lim HC, Castro IP, Hoxey RP. Bluff bodies in deep turbulent boundary layers: Reynolds-number issues. Journal of Fluid Mechanics 2007; 571: 97-118. DOI:10.1017/S00222112006003223.

25. Iyengar AKS, Farell C. Experimental issues in atmospheric boundary layer simulations: roughness length and integral length scale determination. Journal of Wind Engineering and Industrial Aerodynamics 2001; 89(11-12): 1059 1080. DOI: 10.1016/S0167-6105(01)00099-X.

26. Snyder WH, Castro IP. The critical Reynolds number for rough-wall boundary layers. Journal of Wind Engineering and Industrial Aerodynamics 2002; 90: 41-54. DOI:10.1016/S0167-6105(01)00114-3. 
27. Røkenes K, Krogstad PA. Wind tunnel simulation of terrain effects on wind farm siting. Wind Energy 2008; 12: 391-410. DOI:10.1002/we.310.

28. Bakken OM, Krogstad P-A, Ashrafian A, Andersson HI. Reynolds number effects in the outer layer of the turbulent flow in a channel with rough walls. Physics of Fluids 2005; 17(2): 1. DOI:10.1063/1.1900146.

29. DeGraaff D, Eaton JK. Reynolds-number scaling of the flat-plate turbulent boundary layer. Journal of Fluid Mechanics 2000; 422: 319-346. DOI: 10.1017/S0022112000001713.

30. ESDU-85020. Characteristics of atmospheric turbulence near the ground part II: single point data for strong winds (neutral atmosphere), 1985. With amendments A to G August 2001.

31. Yeow TS, Cuerva A, Pérez J. Pressure measurements of the detachment bubble on the Bolund island, International workshop on physical modeling of flow and dispersion phenomena, Hamburg, August 22-24, 2011; 351-360.

32. Bechmann A. Presentations from "The Bolund Experiment: Workshop" 3-4 ${ }^{\text {th }}$ December 2009. Technical Report Ris $\phi-R-1745(E N)$, Risø, 2010.

33. Bradshaw P, Wong FYF. The reattachment and relaxation of a turbulent shear layer. Journal of Fluid Mechanics 1972; 52: 113-135. DOI: $10.1017 /$ S002211207200299X.

34. Yeung CP, Squire LC. Numerical calibration and verification tests of an orthogonal triple-hot-wire probe. Measurement Science and Technology 1993; 4(12): 1446-1456. DOI:10.1088/0957-0233/4/12/022.

35. Bruun HH. Hot-Wire Anemometry. Oxford University Press: New York, 1995. 